\title{
Phenome-wide and genome-wide analyses of quality of life in schizophrenia
}

Raha Pazoki*, Bochao Danae Lin*, Kristel R. van Eijk, Dick Schijven, Sonja de Zwarte, GROUP Investigatorst, Sinan Guloksuz and Jurjen J. Luykx

\section{Background}

Schizophrenia negatively affects quality of life (QoL). A handful of variables from small studies have been reported to influence QoL in patients with schizophrenia, but a study comprehensively dissecting the genetic and non-genetic contributing factors to QoL in these patients is currently lacking.

\section{Aims}

We adopted a hypothesis-generating approach to assess the phenotypic and genotypic determinants of QoL in schizophrenia.

\section{Method}

The study population comprised 1119 patients with a psychotic disorder, 1979 relatives and 586 healthy controls. Using linear regression, we tested $>100$ independent demographic, cognitive and clinical phenotypes for their association with QoL in patients. We then performed genome-wide association analyses of QoL and examined the association between polygenic risk scores for schizophrenia, major depressive disorder and subjective wellbeing and QOL.

\section{Results}

We found nine phenotypes to be significantly and independently associated with QoL in patients, the most significant ones being negative $\left(\beta=-1.17\right.$; s.e. $\left.0.05 ; P=1 \times 10-83 ; r^{2}=38 \%\right)$, depressive $\left(\beta=-1.07\right.$; s.e. $\left.0.05 ; P=2 \times 10-79 ; r^{2}=36 \%\right)$ and emotional distress $\left(\beta=-0.09\right.$; s.e. $\left.0.01 ; P=4 \times 10-59, r^{2}=25 \%\right)$ symptoms.
Schizophrenia and subjective well-being polygenic risk scores, using various $P$-value thresholds, were significantly and consistently associated with QoL (lowest association $P$-value $=6.8 \times$ 10-6). Several sensitivity analyses confirmed the results.

\section{Conclusions}

Various clinical phenotypes of schizophrenia, as well as schizophrenia and subjective well-being polygenic risk scores, are associated with QoL in patients with schizophrenia and their relatives. These may be targeted by clinicians to more easily identify vulnerable patients with schizophrenia for further social and clinical interventions to improve their QoL.

\section{Keywords}

Quality of life; schizophrenia; phenome; genome; polygenic.

\section{Copyright and usage}

(c) The Author(s), 2020. Published by Cambridge University Press on behalf of the Royal College of Psychiatrists. This is an Open Access article, distributed under the terms of the Creative Commons Attribution-NonCommercial-NoDerivatives licence (http://creativecommons.org/licenses/by-nc-nd/4.0/), which permits non-commercial re-use, distribution, and reproduction in any medium, provided the original work is unaltered and is properly cited. The written permission of Cambridge University Press must be obtained for commercial re-use or in order to create a derivative work.
Patients with schizophrenia often experience adverse outcomes, such as unemployment, frequent hospital admissions, long-term dependency on healthcare and suicide. Premature mortality of patients with schizophrenia has been reported to be 3.5 times $^{1}$ greater than that of adults in the general population. The societal costs of schizophrenia during a 12-month period have been estimated to be as high as $\$ 890$ million in the USA. ${ }^{2}$ All domains of quality of life (QoL; physical, psychological and social) are severely decreased in schizophrenia compared with healthy controls. QoL is also increasingly becoming an important index for effectiveness of treatment in schizophrenia. ${ }^{3}$ Several variables have been shown to be associated with QoL among patients with schizophrenia, such as age, gender, employment status, marital status, duration of illness, body mass index, antipsychotic medication, number of hospital admissions, level of knowledge about schizophrenia, schizophrenia symptoms, coping mechanisms and comorbid depression. $^{4-12}$ However, a comprehensive, large-scale study that uses in-depth phenotyping to investigate factors associated with QoL in schizophrenia in a hypothesis-generating fashion is lacking. In addition, to the best of our knowledge, the genetic underpinnings of QoL have not been investigated. Recently published genome-wide association studies (GWASs) for schizophrenia ${ }^{13}$ and related traits, such as major depressive disorder $(\mathrm{MDD})^{14}$

* These authors contributed equally to this work. † Members of the GROUP Investigators, including all affiliations, are available as supplementary material at https://doi.org/10.1192/bjo. 2020.140 and subjective well-being, ${ }^{15}$ provide a timely opportunity to investigate whether genetic mechanisms are at the root of QoL. Knowledge of clinical and genetic contributing factors to QoL in schizophrenia could inform clinicians to help identify vulnerable patients and optimise secondary preventive care, thus reducing the burden of disease. This could be achieved through optimisation of treatment regimens (e.g. psychosocial interventions or optimising psychopharmacological treatments) and targeting clinical variables negatively influencing QoL. On a similar note, insight into genetic factors contributing to QoL could contribute to the early identification of vulnerable patients, and improve their outcome.

Here, we used a hypothesis-generating approach and investigated over 100 phenotypes to investigate factors related to QoL among patients with schizophrenia. We additionally performed genetic risk scoring in patients, relatives and healthy controls to uncover associations between genetic susceptibility to schizophrenia, MDD and subjective well-being on the one hand, and QoL on the other.

\section{Method}

\section{Participants and study design}

All procedures contributing to this work comply with the ethical standards of the relevant national and institutional committees on human experimentation and with the Helsinki Declaration of 1975, as revised in 2008. All procedures involving human 
subjects/patients were approved by the medical-ethical committee of University Medical Center Utrecht. All participants provided written informed consent for the study. The current study was performed within a cohort of 3684 individuals, including 1119 patients with schizophrenia, 1059 siblings, 920 parents and 586 controls (Supplementary Appendix 2, Supplementary Fig. 1 available at https://doi.org/10.1192/bjo.2020.140). We used two main subsets. The first subset included patients only $(n=1119)$, to test nongenetic contributing factors to QoL among patients with schizophrenia. We chose this subset as we were interested in phenotypic contributing factors to QoL in patients; however, other, larger cohort studies may be more appropriate to probe contributing factors to QoL in the general population. The second subset included patients, relatives and controls with genetic data available $(n=2265)$, to test genetic contributing factors to QoL. We chose this subset to increase statistical power and as, intuitively, genetic contributing factors to QoL may (partly) overlap between patients, relatives and controls. All participants were included from the Genetic Risk and Outcome of Psychosis (GROUP) study, ${ }^{16}$ a multicentre, large longitudinal study in The Netherlands and Belgium, investigating various psychological and genetic variables among patients with schizophrenia and their relatives. The study population was followed up since 2004, in several mental healthcare institutions, both in The Netherlands and Belgium. The detailed phenotypic information of GROUP participants offers a unique and enriched database. ${ }^{16}$ Psychosis-related and demographic variables that were included in the analysis are presented in Supplementary Appendix 1. These variables may be divided into symptoms and experiences that were assessed with a range of (semi)-structured scales, including drug use, family loading for psychiatric disorders, social cognition, demographic variables, IQ, medication use data and theory of mind scales. For the purpose of this study, we only used the baseline assessments of the GROUP study (release 5.00), as we were interested in factors contributing to QoL in schizophrenia apparent in its early disease stages (the first psychotic episode of the GROUP participants had to occur within 10 years before this first assessment). Supplementary Figure 1 shows a breakdown of the study sample.

\section{QoL assessment}

QoL was assessed with the World Health Organization's WHOQOL-BREF, an abbreviated version of the World Health Organization Quality of Life scale. The self-report WHOQOLBREF has been validated for a Dutch-speaking population of psychiatric patients. ${ }^{16-18}$ Details of the Dutch WHOQOL-BREF are described elsewhere. $^{18}$ The WHOQOL-BREF scale includes 24 items covering four domains of QoL, ${ }^{18}$ namely physical health (seven items), psychological (six items), social relations (three items) and environment (eight items). The questionnaire additionally provides a general estimate of QoL presented as five categories: 'very bad', 'moderately bad', 'good nor bad', 'moderately good' and 'very good'. To reduce the number of QoL variables and create a suitable variable for statistical analyses, we performed principal component analysis to preferably identify one quantitative component that best explained most of the variability in QoL domains, without transformations, only in patients. Similar to a previously published GWAS using principal component analysis to derive a single phenotype, ${ }^{19}$ the percentage of variance accounted for by the first unrotated principal component was computed. This principal component was standardised for further analysis, explained $62.5 \%$ of the variability in our data and correlated well with all WHOQOL-BREF domains (Supplementary Fig. 2), whereas the other four components were correlated with only one of the domains and showed minimal correlations with other domains of
QoL. We therefore used this first principal component for subsequent analyses.

\section{Phenome-wide analyses}

For the agnostic association analysis of QoL with the above explained demographic and clinical phenotypes (Supplementary Appendix 1), we used data from patients with complete data on the QoL principal component, age, gender and study site $(n=925$; Supplementary Fig. 1). The number of independent variables was calculated by testing two-by-two correlations between variables, using non-parametric Spearman correlation. Variables with correlation estimates $>0.3$ and statistically significant correlations $(P<0.05)$ were considered interdependent variables. This analysis resulted in 105 independent variables. Generalised linear models (GLM) adjusted for age, gender and study site were then used to test the association of QoL with each of the clinical phenotypes. The statistical significance threshold for this association analysis was corrected for multiple testing with the Bonferroni correction method, i.e. $4.76 \times 10^{-04}(0.05$ adjusted for 105 independent tests). ${ }^{20}$ As sensitivity analysis, we calculated $P$-values for phenotype-QoL associations by using data with random samples of the QoL variable, using 1000 iterations. We then calculated the probability that our observed $P$-values are driven by chance (empirical $P$-values) by using the method of Davison and Hinkley, ${ }^{21}$ as follows: (number of null $P$-values less than the observed +1 )/ (number of permutations +1 ). For each trait-QoL association, we declared statistical significance if none of the 1000 iterated samples led to a permutated $P$-value less than the observed $P$-value. This was equivalent to an empirical $P$-value of 0.001 . The variance in QoL explained by the phenotypes was calculated with $\mathrm{R}^{2}$ values obtained from GLM. To then identify a set of variables that were associated with QoL independent of one another, we used the phenotypes that were associated with QoL at $P<4.76 \times 10^{-04}$ and selected the independent variables in a backward stepwise regression model. As a sensitivity analysis, we then regressed the most significantly associated phenotypes with ordinal estimates of QoL as opposed to the first principal component of QoL.

\section{Genotyping, quality control and genome-wide association analysis}

Details of genotyping and GWAS of QoL can be found in Supplementary Appendix 1. In brief, genotype data for 2812 GROUP participants were generated on a customised Illumina Institute of Psychological Medicine and Clinical Neurology chip array with 570038 single-nucleotide polymorphisms (SNPs). Quality control procedures were performed with PLINK, version 1.9 for Apple Mac (Harvard, USA; see https://zzz.bwh.harvard. edu/plink/download.shtml). ${ }^{22}$ In total, 2505 individuals and 275021 SNPs passed these abovementioned quality control steps. After merging with the phenotype file, 2265 individuals were left for genetic analyses (Supplementary Appendix 2, Supplementary Fig. 1).

Additional SNPs were imputed on the Michigan server, ${ }^{23}$ using the Haplotype Reference Consortium r1.1 2016 reference panel. Although likely underpowered, for the benefit of possible future meta-analyses and as a first exploratory approach we performed linear mixed models association testing implemented in BOLTLMM (version 2.3) software for Apple Mac (Broad Institute, USA; see https://alkesgroup.broadinstitute.org/BOLT-LMM/BOLTLMM_manual.html $)^{24}$ to assess associations between SNPs and QoL (Supplementary Appendix 1). BOLT-LMM corrects for confounding from population structure and cryptic relatedness. We used the generally accepted association $P$-value threshold of $P<5 \times 10^{-8}$ 
for genome-wide significance. We report those findings in the Supplementary Appendix 2 (Supplementary Figs. 7 and 8).

\section{Polygenic risk score analyses of QoL}

We used recent GWASs of schizophrenia, ${ }^{13} \mathrm{MDD}^{14}$ and subjective well-being ${ }^{15}$ for polygenic risk score (PRS) calculations. ${ }^{25}$ We chose the PRSs of these disorders as they are strongly associated with QoL in the general population. ${ }^{26-29}$ To verify that PRS of other traits were indeed unlikely to be associated with QoL, the genetic correlations between the primary BOLT- LMM GWAS summary statistics and over 700 other disease traits were estimated with linkage disequilibrium score regression (http://ldsc.broadinstitute.org/). ${ }^{30}$ As a quality control for PRS calculation, the SNPs that overlapped between the summary statistics GWASs (training data-sets) and our data-set ${ }^{31}$ were extracted. Then, insertions or deletions, ambiguous SNPs, SNPs with minor allele frequency $<0.01$ and imputation quality $\left(\mathrm{R}^{2}\right)<0.8$ in both training and target data-sets were excluded. To account for complicated linkage disequilibrium structure of SNPs in the genome, these SNPs were clumped in two rounds with PLINK version $1.90 \mathrm{~b} 3 \mathrm{z},{ }^{32}$ according to previously established methods: ${ }^{33,34}$ round 1 with the default parameters (physical distance threshold $250 \mathrm{~kb}$ and linkage disequilibrium threshold $\left(\mathrm{R}^{2}\right)$ of 0.5 ); and round 2 with a physical distance threshold of $5000 \mathrm{~kb}$ and linkage disequilibrium threshold $\left(\mathrm{R}^{2}\right)$ of 0.2 . Additionally, we excluded all SNPs in genomic regions with strong or complex linkage disequilibrium structures (e.g. the MHC region on chromosome 6; Supplementary Appendix 2, Supplementary Table 1). If only odds ratios were reported in the summary statistics, they were logconverted to $\beta$-values as effect sizes. To prevent possible study population overlap affecting our results, all Dutch and Belgian individuals had been excluded from the schizophrenia GWAS ${ }^{13}$ to allow unbiased PRS computation. ${ }^{33}$ Sample overlap between GROUP data with MDD and subjective well-being GWAS samples is unlikely because all samples belong to different cohorts. To reassure that there was indeed minimal to no sample overlap between GROUP and MDD and subjective well-being samples, we checked the intercepts of the genetic covariances from linkage disequilibrium score regression analyses between the GROUP GWAS and MDD and subjective well-being. Presence of sample overlap modifies this intercepts from zero, ${ }^{35}$ whereas in our study all intercepts turned out to be close to zero (Supplementary Appendices 1 and 2, Supplementary Table 2). We constructed PRSs based on schizophrenia risk alleles weighted by their schizophrenia-increasing effect estimate, using the Purcell et al method, ${ }^{22,36}$ i.e. using PLINK's score function for 12 GWAS $P$-value thresholds (referred to as $P$ t from here onward): $5 \times 10^{-8}, 5 \times 10^{-7}, 5 \times 10^{-6}, 5 \times 10^{-5}, 5 \times 10^{-4}, 5 \times 10^{-3}$, $0.05,0.1,0.2,0.3,0.4$ and 0.5 . PRSs were calculated for 2505 patients, relatives and controls (those remaining after quality control). Genetic data and QoL variables were available for patients, relatives and controls. We thus performed statistical analyses for the association of PRSs with QoL in the whole sample after quality control
( $N=2265$; Supplementary Appendix 2, Supplementary Fig. 1), including patients, controls and family members. This approach provided the opportunity to investigate genetic susceptibility of these PRSs on QoL regardless of presence or absence of the disease. We calculated explained variance and $P$-values for PRSs on QoL in two stages. In the first stage, we analysed the effect of age, gender and disease status on QoL to obtain residuals of this model. Subsequently, we tested the association of the residuals of the previous model with each of the various PRSs, using a linear mixed model with family identification as random effect and the first three genetic principal components as covariates. We additionally included the most significantly associated PRSs from each schizophrenia, MDD and well-being PRS group in one single mixed model, to assess if these PRSs were statistically independent of each other. We subsequently included negative, positive and depressive symptoms as covariates (as these were previously known to associate with QoL, which was also confirmed in the current cohort), and PRSs in mixed models to assess the additional explained variance of the three PRSs beyond clinical phenotypes. We additionally performed sensitivity schizophrenia PRS analyses on patients only, to assess whether we observed similar effects to the combined set of patients, siblings and controls.

To claim significance for association analyses between PRS and QoL, we Bonferroni-corrected the $P$-value for multiple testing ( $0.05 /$ $3=0.016$ ), which is likely to be conservative given the significant and sizeable genetic correlations between the three PRS traits tested.

\section{Results}

Table 1 shows baseline characteristics of patients, relatives and controls. In the GLM, 18 distinct variables were associated with QoL at the Bonferroni significance threshold of $4.76 \times 10^{-04}$ in patients with schizophrenia (Fig. 1, Table 2 and Supplementary Appendix 2, Supplementary Fig. 3). In our permutation sensitivity analysis, the same variables were significantly associated with QoL in addition to one extra variable, educational degree (Table 2). The statistically most significant phenotypes were negative $(\beta=-1.17$; s.e. $0.05, P=1 \times 10^{-83} ; r^{2}$ model $\left.=38 \%\right)$, depressive $(\beta=-1.07$; s.e. 0.05 ; $P=2 \times 10^{-79} ; r^{2}$ model $\left.=36 \%\right)$, emotional distress $(\beta=-0.09$; s.e. $0.01 ; P=4 \times 10^{-59} ; r^{2}$ model $\left.=25 \%\right)$ and general psychopathology $\left(\beta=0.81 ;\right.$ s.e. $0.06 ; \quad P=3 \times 10^{-40} ; r^{2}$ model $\left.=17 \%\right)$ symptoms (Table 2). In our regression model including these 18 variables, nine remained independently associated with QoL $(P<0.05)$, explaining $58.55 \%$ of the variance in QoL. Ordered by decreasing level of significance these are: negative symptoms, global assessment of functioning, emotional distress, depressive symptoms, positive symptoms, remission status, cannabis craving, number of unmet needs and excitement (Table 2 and Supplementary Appendix 2, Supplementary Table 3 ). In addition, there was a negative age effect $\left(\beta=-0.01\right.$; s.e. $\left.0.003 ; P=3 \times 10^{-3}\right)$ on QoL in the backward stepwise model (Supplementary Appendix 2, Supplementary

Table 1 Baseline characteristics for patients, siblings and controls

\begin{tabular}{|c|c|c|c|c|}
\hline Characteristics & Patients $(n=1119)$ & Controls $(n=586)$ & Siblings $(n=1059)$ & Parents $(n=920)$ \\
\hline Age in years, mean (s.d.) & $27.6(7.9)$ & $30.4(10.6)$ & $27.8(8.3)$ & $54.7(6.7)$ \\
\hline Gender, $n(\%)$ women & $267(23.9)$ & $317(54.1)$ & $577(54.5)$ & $528(57.4)$ \\
\hline IQ, estimated, mean (s.d.) & $95(16.1)$ & $109.7(15.1)$ & $102.8(15.6)$ & $103(17.0)$ \\
\hline Married/living together, $n(\%)$ & $97(9.3)$ & $234(41.1)$ & $411(40.2)$ & $153(70.8)$ \\
\hline Years of education, mean (s.d.) & $4(2.1)$ & $5.4(1.8)$ & $5.1(2.1)$ & $5.1(2.3)$ \\
\hline Nicotine use, mean number of cigarettes daily (s.d.) & $11.7(11)$ & $3(6.5)$ & $4.9(8.4)$ & $4.3(8.8)$ \\
\hline Alcohol use, mean number of drinks per week (s.d.) & $6.6(12.1)$ & $6.1(8.5)$ & $6.4(8.6)$ & $8.1(10.6)$ \\
\hline Current use of antipsychotics, $n(\%)$ & $1062(95)$ & $0(0)$ & $0(0)$ & $2(0.22)$ \\
\hline Duration of illness (years), mean (s.d.) & $4.2(4)$ & Not applicable & Not applicable & Not applicable \\
\hline
\end{tabular}




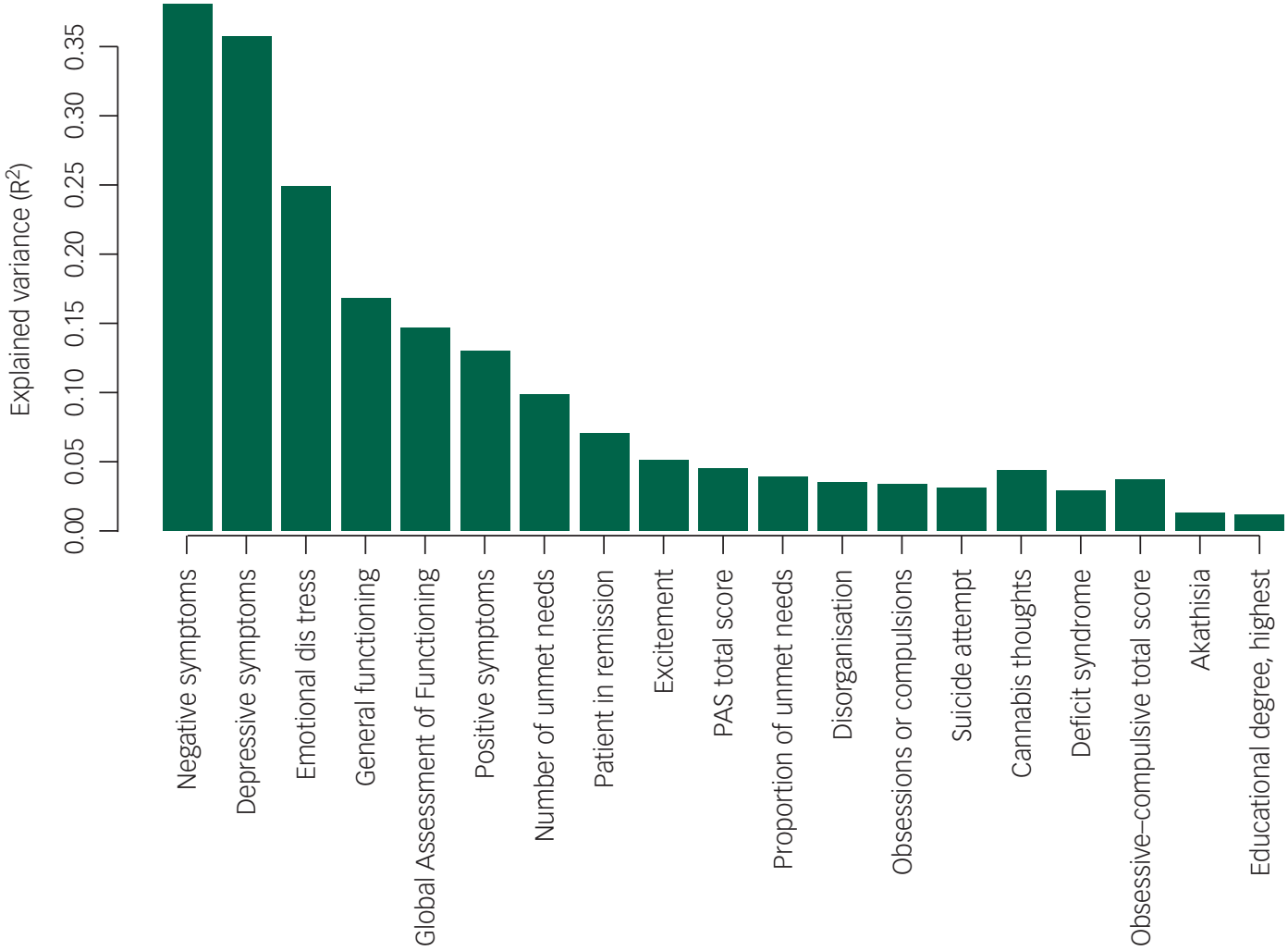

Fig. 1 Results of the hypothesis-generating association analysis between clinical variables and QoL among patients with schizophrenia with explained variance for QOL. Number of unmet needs was measured with the Camberwell Assessment of Need. Remission was measured with the PANSS patient in remission tool. Suicide attempt was assessed with the composite file (a questionnaire with closed questions designed for the Genetic Risk and Outcome of Psychosis study). Cannabis thoughts were defined as thoughts about cannabis use, measured with the Obsessive Compulsive Drug Use Scale. Deficit syndrome was measured with the Schedule for the Deficit Syndrome. Obsessive-compulsive symptoms total score was measured with the Yale-Brown Obsessive Compulsive Scale. Akathisia was measured with the Barnes akathisia rating scale. PAS, Premorbid Adjustment Scale.

\begin{tabular}{|c|c|c|c|c|c|c|}
\hline Variable & Scale & $\begin{array}{l}\text { Standardised } \\
\text { effect estimate }\end{array}$ & s.e. & $\begin{array}{l}\text { Explained } \\
\text { variance }\end{array}$ & $P$-value & $\begin{array}{c}\text { Empirical } \\
P \text {-value }\end{array}$ \\
\hline Negative symptoms, points & CAPE & -1.17 & 0.05 & 0.38 & $1 \times 10^{-83}$ & 0.001 \\
\hline Depressive symptoms, points & CAPE & -1.07 & 0.05 & 0.36 & $2 \times 10^{-79}$ & 0.001 \\
\hline Emotional distress, points & PANSS & -0.09 & 0.01 & 0.25 & $4 \times 10^{-59}$ & 0.001 \\
\hline General psychopathology symptoms, points & PANSS & -0.81 & 0.06 & 0.17 & $3 \times 10^{-40}$ & 0.001 \\
\hline Global Assessment of Functioning (disabilities), points ${ }^{a}$ & GAF & 0.03 & 0 & 0.15 & $2 \times 10^{-34}$ & 0.001 \\
\hline Positive symptoms, points & CAPE & -0.05 & 0 & 0.13 & $1 \times 10^{-23}$ & 0.001 \\
\hline Number of unmet needs, points & CAN & -0.11 & 0.01 & 0.10 & $3 \times 10^{-23}$ & 0.001 \\
\hline Remission status, yes & PANSS & -0.56 & 0.07 & 0.07 & $8 \times 10^{-17}$ & 0.001 \\
\hline Excitement, points & PANSS & -0.06 & 0.01 & 0.05 & $9 \times 10^{-13}$ & 0.001 \\
\hline PAS total score & PAS & -0.25 & 0.04 & 0.04 & $5 \times 10^{-11}$ & 0.001 \\
\hline Proportion of unmet needs & CAN & -0.69 & 0.11 & 0.04 & $2 \times 10^{-9}$ & 0.001 \\
\hline Disorganisation, points & PANSS & -0.03 & 0.01 & 0.04 & $3 \times 10^{-09}$ & 0.001 \\
\hline Obsessive-compulsive symptoms, yes & Y-BOCS & -0.43 & 0.08 & 0.03 & $4 \times 10^{-08}$ & 0.001 \\
\hline Suicidal attempts (lifetime), yes & Composite file ${ }^{b}$ & -0.43 & 0.08 & 0.03 & $6 \times 10^{-08}$ & 0.001 \\
\hline Cannabis craving, yes & OC-DUS & -0.31 & 0.06 & 0.04 & $1 \times 10^{-07}$ & 0.001 \\
\hline Obsessive-compulsive total score & OC-DUS & -0.28 & 0.06 & 0.04 & $1 \times 10^{-06}$ & 0.001 \\
\hline Deficit syndrome & SDS & -0.39 & 0.08 & 0.03 & $1.19 \times 10^{-06}$ & 0.001 \\
\hline Akathisia & BARS & -0.15 & 0.04 & 0.01 & $3 \times 10^{-04}$ & 0.001 \\
\hline Educational degree & & 0.06 & 0.02 & 0.01 & $5 \times 10^{-04 c}$ & 0.001 \\
\hline
\end{tabular}


Table 3). Association analysis between ordinal estimates of QoL showed similar results (Supplementary Appendix 2, Supplementary Fig. 4). We additionally tested if any of these phenotypes captured in the original World Health Organization questionnaire items showed associations with the phenotypes tested, and we observed that the World Health Organization mobility item explained $32 \%, 25 \%, 21 \%$ and $9 \%$ of the variances in depressive, negative, positive and emotional symptoms, respectively. The rest of the World Health Organization items each explained less than $8 \%$ variation in each of our statistically most significant phenotypes.

The variance in QoL explained by various PRSs $(N=2265)$ were $1.37 \%$ for schizophrenia, $1.37 \%$ for subjective well-being (Fig. 2) and $1.40 \%$ for MDD (Supplementary Appendix 2, Supplementary Fig. 5) when using only genome-wide significant SNPs (Pt of $5 \times 10^{-8}$ ). The most significant associations between PRS and QoL were observed for schizophrenia $\left(\mathrm{Pt}_{0.5}\right.$; explained variance $1.58 \%, P=$ $7 \times 10^{-6}$; Fig. 1), subjective well-being $\left(\mathrm{Pt}_{0.4}\right.$; explained variance $1.82 \%, P=0.004$; Fig. 1$)$ and $\mathrm{MDD}\left(\mathrm{Pt}_{0.005}\right.$; explained variance $1.62 \%, \quad P=0.01$; Supplementary Appendix 2, Supplementary Fig. 5). As a sensitivity analysis, we repeated the schizophrenia PRS analysis on patients only $(n=633)$, and confirmed the same pattern of association with QoL and the same Pt of 0.5 showing most significant association results (Supplementary Appendix 2, Supplementary Fig. 6). The SNP heritability of QoL was 13.19\% (s.e. 19.03). As expected, given the relatively low statistical power, genetic correlation analysis in LD Hub showed no statistically significant results. Confirming our rationale for investigating the PRSs chosen in the current study, genetic correlations of QoL with schizophrenia $^{13}$ and subjective well-being ${ }^{15}$ were the strongest, in the expected direction (Supplementary Appendix 2, Supplementary Table 2).
Schizophrenia $\mathrm{Pt}_{0.5}\left(P=7 \times 10^{-6}\right), \mathrm{MDD} \mathrm{Pt}_{0.005}(P=0.01)$ and subjective well-being $\mathrm{Pt}_{0.4}(P=0.004)$ remained associated with QoL independent of one another. After additional adjustment for positive, negative and depressive symptoms, schizophrenia PRS $\left(\mathrm{Pt}_{0.5} ; P=0.002\right)$ and well-being $\mathrm{PRS}\left(\mathrm{Pt}_{0.4} ; P=0.04\right)$ remained associated with QoL. Moreover, only schizophrenia and subjective well-being PRSs were consistent with true polygenicity, explaining a proportion of the variance in QoL, as may be appreciated by increasing degrees of explained variances and increasing significance levels with relaxing Pts (Fig. 2).

As stated above, all final clinical phenotypes included in our regression model together explained $58.55 \%$ of the variability in QoL. By adding schizophrenia $\mathrm{Pt}_{0.5}, \mathrm{MDD} \mathrm{Pt}_{0.005}$ and subjective well-being $\mathrm{Pt}_{0.4}$, the model explained $59.00 \%$ of the variability.

\section{Discussion}

Here, we identify non-genetic factors contributing to QoL among patients suffering from schizophrenia. Our results show that $\sim 58 \%$ of variance in QoL may be explained by a range of demographic and clinical variables. We additionally demonstrate that genetic predisposition to schizophrenia and subjective well-being explain a (small) proportion of variability in QoL on top of clinical variables. The novelty of our method lies in the use of hypothesisgenerating approaches to investigate a vast number of schizophrenia-associated genetic and non-genetic variables.

Most of the previous studies into QoL in schizophrenia had small-to-moderate sample sizes, ${ }^{10,11,37}$ and have shown the association of particularly negative and positive symptoms with QoL in schizophrenia. A recent study in 157 patients with schizophrenia

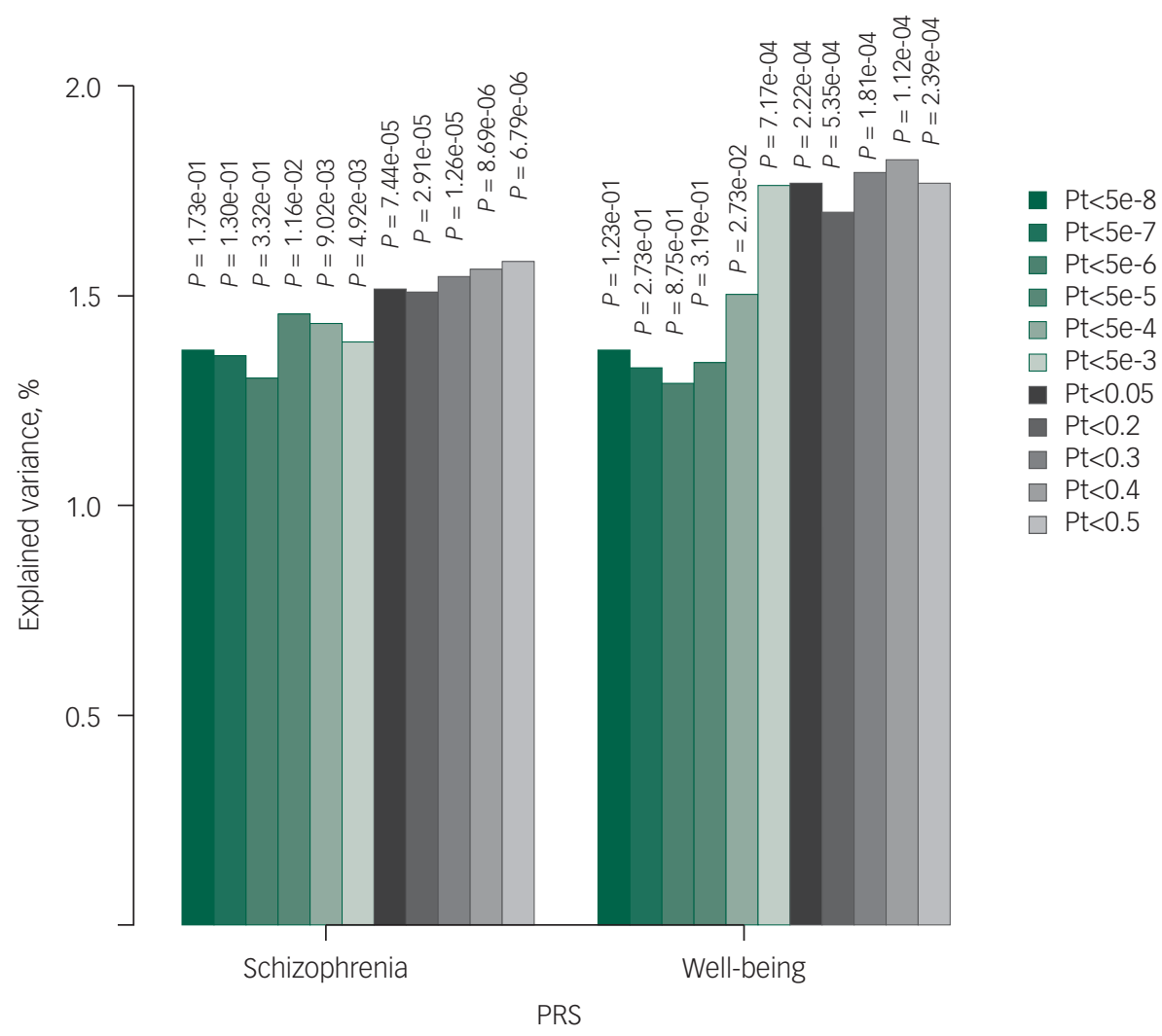

Fig. 2 Bar plot illustrating explained variance for association of polygenic risk scores of schizophrenia and subjective well-being with quality of life. The figure illustrates the results with linear mixed models. Displayed are the number of single-nucleotide polymorphisms $(N)$, the strengths of the association results $\left(-\log _{10} P\right.$-value) and explained variances per Pt ( $P$-value threshold). PRS, polygenic risk score. 
showed the effects of excitement, ${ }^{29}$ positive, negative and depres$\operatorname{sive}^{29}$ symptoms on QoL. The study by Domenech et al used only clinical symptoms based on PANSS. ${ }^{29}$ We tested clinical symptoms assessed with multiple internationally well-established scales (e.g. Community Assessment of Psychic Experience, Positive And Negative Syndrome Scale, Camberwell Assessment of Need) and assessed a range of other phenotypic variables. Such rich phenotyping together with our large sample size allowed us to firmly establish additional variables associated with QoL in schizophrenia at increased statistical significance. Moreover, this approach allowed us to weigh the effect of all variables in one model. In line with our findings, a recent meta-analysis also found a substantial association between depressive symptoms and personal recovery, a concept related to QoL. ${ }^{38}$

Several of the variables we found to be associated with QoL in schizophrenia had, to the best of our knowledge, not been reported, such as disorganisation, obsessive-compulsive symptoms, suicidal attempts, unmet needs, akathisia and cannabis craving. Although the underlying mechanisms of this latter association are still unclear, one may speculate that cannabis craving constitutes a proxy for cannabis abstinence, which in turn may increase anxiety and thus reduce psychological well-being. Alternatively, relatively high levels of cannabis dependence may worsen symptoms and thus negatively affect QoL.

Genetic predisposition to schizophrenia captured by PRS showed clear and persistent effects on QoL across all Pts. We also observed moderate effects of polygenic susceptibility to subjective well-being on QoL and no independent effects of genetic predisposition to MDD on QoL in our cohort. Intriguingly, although statistical power for PRSs derived from large GWAS cohorts is usually relatively large, we found stronger associations for schizophrenia PRS (GWAS N=36989 cases and 113075 controls) than for MDD PRS (GWAS N=135458 cases and 344901 controls) and subjective well-being PRS (GWAS $N=298420$ ). Possibly, genetic architecture (relatively large SNP-based heritability for schizophrenia) and case-control ascertainment (relatively low numbers of incorrectly diagnosed controls in schizophrenia) explain a stronger association signal for schizophrenia PRS with QoL. Alternatively, QoL in schizophrenia may simply be more genetically related to schizophrenia than MDD, given how the nature of the disease affects QoL in people who suffer from it. Hypothetically, MDD and subjective well-being PRSs may be stronger determinants of QoL in people who do not suffer from schizophrenia.

The current study benefits from a large sample size of a multicentre, prospective cohort study in The Netherlands, with comprehensive phenotypic assessments in individuals with schizophrenia. The large sample size increases precision and reliability of our findings. The combination of a large sample size and rich phenotyping created a unique opportunity for a phenome-wide study to identify contributing factors to QoL in schizophrenia. In addition, carefully selected participants from several geographical locations restricted the risk of selection bias. On the other hand, several limitations should be borne in mind when interpreting our results. First, interpretation of principal component-driven variables may not be intuitive. Here, we managed to show its feasibility and usefulness. We reduced the number of variables of the four different domains of QoL into one variable and were able to assess the impact of multiple clinical and genetic determinants on this variable. Our results showed consistency in terms of direction and magnitude of the effect estimate when compared with ordinal domains of QoL (Supplementary Fig. 3). Second, we are aware of the relatively low power for genetic studies on a complex trait such as QoL, both in our GWAS and linkage disequilibrium score regression analyses. Our GWAS must therefore be regarded as a first exploratory GWAS of QoL in patients with schizophrenia, their siblings and healthy controls. Similarly, for linkage disequilibrium score regression, we were underpowered to reveal clear genetic correlations. Linkage disequilibrium score regression analysis was done to explore possible genetic correlations with traits different from the ones we investigated, and to investigate whether the trait with most significant genetic correlation results was identical to the trait with most significant PRS results, which indeed turned out to be the case. Third, in the study population, about $97 \%$ of participants were White, which hampers generalisability to other ethnicities. Fourth, given the uniqueness of our cohort and the lack of QoL phenotyping data in other cohorts, we were unable to perform replication analyses. We encourage researchers to collect QoL and genome-wide data to allow for such replication efforts. Finally, our association analyses preclude us from drawing definite conclusions about causality. Future, well-powered, prospective studies are necessary to improve insight into possible causal mechanisms.

In conclusion, we highlight multiple clinical and genetic associations with QoL that could be leveraged in daily care of patients with schizophrenia to improve their QoL. The variables highlighted in the current study could aid health professionals who interact with patients with psychosis to more readily recognise the need for additional interventions in patients showing a high burden of such phenotypes. For example, although high levels of positive and negative symptoms are intuitively associated with QoL, disorganisation, cannabis craving and obsessive-compulsive symptoms are also important contributors, according to our analyses. Genetic risk scoring may furthermore be used to optimise identification of those patients with schizophrenia who are susceptible to low QoL, which in turn may advance timely management for these vulnerable patients.

Raha Pazoki, MD, PhD, Department of Psychiatry, Brain Center Rudolf Magnus, University Medical Center Utrecht, Utrecht University, Utrecht, The Netherlands; and Department of Epidemiology, Imperial College London, School of Public Health, UK; Bochao Danae Lin, PhD, Department of Translational Neuroscience, Brain Center Rudolf Magnus, University Medical Center Utrecht, Utrecht University, Utrecht, The Netherlands; Kristel R. van Eijk, PhD, Department of Translational Neuroscience, Brain Center Rudolf Magnus, University Medical Center Utrecht, Utrecht University, Utrecht, The Netherlands; Dick Schijven, PhD, Department of Psychiatry, Brain Center Rudolf Magnus, University Medical Center Utrecht, Utrecht University, Utrecht, the Netherlands; and Department of Translational Neuroscience, Brain Center Rudolf Magnus, University Medical Center Utrecht, Utrecht University, Utrecht, The Netherlands; Sonja de Zwarte, PhD, Department of Psychiatry, Brain Center Rudolf Magnus, University Medical Center Utrecht, Utrecht University, Utrecht, The Netherlands; GROUP Investigators; Sinan Guloksuz, MD, PhD, Department of Psychiatry and Neuropsychology, Maastricht University Medical Center, School for Mental Health and Neuroscience, Maastricht, The Netherlands; and Department of Psychiatry, Yale University School of Medicine, New Haven, Connecticut, USA; Jurjen J. Luykx (D, MD, PhD, Department of Psychiatry, Brain Center Rudolf Magnus, University Medical Center Utrecht, Utrecht University, Utrecht, The Netherlands; Department of Translational Neuroscience, Brain Center Rudolf Magnus, University Medical Center Utrecht, Utrecht University, Utrecht, The Netherlands; and Outpatient Second Opinion Clinic, GGNet, Warnsveld, The Netherlands

Correspondence: Jurjen Luykx.Email: j.luykx@umcutrecht.nl

First received 10 Jan 2020, final revision 20 Oct 2020, accepted 5 Nov 2020

\section{Supplementary material}

Supplementary material is available online at https://doi.org/10.1192/bjo.2020.140

\section{Data availability}

All authors have continuous access to the data, both phenotypic and genotypic, collected in this study. Summary data are available upon reasonable request.

\section{Author contributions}

All authors were involved in writing the manuscript. Statistical analyses were done by R.P. B.D.L., K.R.V.E., D.S. and S.d.Z. S.G. and J.J.L. supervised this work. J.J.L. and R.P. conceived B.D.L., K.R.V.E., D.S. and S.d.Z. S.G. and J.J.L. supers
the study. J.J.L., R.P. and B.D.L. wrote the first draft.

\section{Funding}

The infrastructure for the GROUP study is funded through the Geestkracht programme of the Dutch Health Research Council (Nederlandse organisatie voor gezondheidsonderzoek en zorginnovatie, grant number 10-000-1001), and matching funds from participating pharmaceutical 
companies (Lundbeck, AstraZeneca, Eli Lilly, Janssen Cilag) and universities and mental healthcare organisations (Amsterdam: Academic PSychiatric Centre of the Academic Medical Center and the Rivierduinen, Erasmus Medical Centre and GGZ Noord Holland Noord; Groningen: University Medical Center Groningen and the mental health institutions at Lentis, GGZ Friesland, GGZ Drenthe, Dimence, Mediant, GGNet Warnsveld, Yulius Dordrecht and Parnassia Psycho-Medical Center The Hague; Maastricht: Maastricht University Medical Centre and the mental health institutions at GGZ Eindhoven en De Kempen, GGZ Breburg, GGZ Oost-Brabant, Vincent van Gogh voor Geesteliike Gezondheid, Mondriaan, Virenze riagg Zuyderland GGZ, MET go Universitair centrum sint-Jozef Maria Sint-Truiden, GGZ Overpelt and Openbaar Psychiatrisch Zorgcentrum Rekem; Utrecht: University Medical Center Utrecht and the mental health institutions Altrecht, GGZ Centraal and Delta).

\section{Declaration of interest}

None

ICMJE forms are in the supplementary material, available online at https://doi.org/10.1192/ bjo.2020.140

\section{References}

1 Olfson M, Gerhard T, Huang C, Crystal S, Stroup TS. Premature mortality among adults with schizophrenia in the United States. JAMA Psychiatry 2015; 72(12): 1172-81.

2 Evensen S, Wisloff T, Lystad JU, Bull H, Ueland T, Falkum E. Prevalence, employment rate, and cost of schizophrenia in a high-income welfare society: a population-based study using comprehensive health and welfare registers. Schizophr Bull 2016; 42(2): 476-83.

3 Kane JM, Robinson DG, Schooler NR, Mueser KT, Penn DL, Rosenheck RA, et al. Comprehensive versus usual community care for first-episode psychosis: 2year outcomes from the NIMH RAISE early treatment program. Am J Psychiat 2016; 173(4): 362-72.

4 Hasan A, Tumah H. Determinants of quality of life among people diagnosed with schizophrenia at outpatient clinics. Perspect Psychiatr C 2019; 55(1): 30-9.

5 Hofer A, Mizuno Y, Wartelsteiner F, Fleischhacker WW, Frajo-Apor B, Kemmler $\mathrm{G}$, et al. Quality of life in schizophrenia and bipolar disorder: the impact of symptomatic remission and resilience. Eur Psychiat 2017; 46: 42-7.

6 Hou CL, Ma XR, Cai MY, Li Y, Zang Y, Jia FJ, et al. Comorbid moderate-severe depressive symptoms and their association with quality of life in Chinese patients with schizophrenia treated in primary care. Community Ment HIt J 2016; 52(8): 921-6.

7 Karow A, Wittmann L, Schottle D, Schafer I, Lambert M. The assessment of quality of life in clinical practice in patients with schizophrenia. Dialogues Clin Neuro 2014; 16(2): 185-95.

8 Rayan A, Obiedate $\mathrm{K}$. The correlates of quality of life among Jordanian patients with schizophrenia. J Am Psychiat Nurses 2017; 23(6): 404-13.

9 Rotstein A, Roe D, Gelkopf M, Levine SZ. Age of onset and quality of life among males and females with schizophrenia: a national study. Eur Psychiat 2018; 53: 100-6.

10 Savill M, Orfanos S, Reininghaus U, Wykes T, Bentall R, Priebe S. The relationship between experiential deficits of negative symptoms and subjective quality of life in schizophrenia. Schizophr Res 2016; 176(2-3): 387-91.

11 Wang $X Q$, Petrini MA, Morisky DE. Predictors of quality of life among chinese people with schizophrenia. Nurs Health Sci 2017; 19(2): 142-8.

12 Yamauchi K, Aki H, Tomotake M, Iga Jl, Numata S, Motoki I, et al. Predictors of subjective and objective quality of life in outpatients with schizophrenia. Psychiat Clin Neuros 2008; 62(4): 404-11.

13 Ripke S, Neale BM, Corvin A, Walters JTR, Farh KH, Holmans PA, et al. Biological insights from 108 schizophrenia-associated genetic loci. Nature 2014; 511 (7510): 421-7.

14 Wray NR, Ripke S, Mattheisen M, Trzaskowski M, Byrne EM, Abdellaoui A, et al. Genome-wide association analyses identify 44 risk variants and refine the genetic architecture of major depression. Nat Genet 2018; 50(5): 668-81.

15 Okbay A, Baselmans BML, De Neve J-E, Turley P, Nivard MG, Fontana MA, et al. Genetic variants associated with subjective well-being, depressive symptoms, and neuroticism identified through genome-wide analyses. Nat Genet 2016; 48: 624.

16 Korver N, Quee PJ, Boos HB, Simons CJ, de Haan L. Genetic Risk and Outcome of Psychosis (GROUP), a multi-site longitudinal cohort study focused on gene- environment interaction: objectives, sample characteristics, recruitment and assessment methods. Int J Methods Psychiatr Res 2012; 21(3): 205-21.

17 Trompenaars FJ, Masthoff ED, Van Heck GL, Hodiamont PP, De Vries J. Conten validity, construct validity, and reliability of the WHOQOL-Bref in a population of Dutch adult psychiatric outpatients. Qual Life Res 2005; 14(1): 151-60.

18 Gobbens RJJ, van Assen MALM. Psychometric properties of the Dutch WHOQOL-OLD. Health Qual Life Outcomes 2016; 14: 103.

19 Davies G, Lam M, Harris SE, Trampush JW, Luciano M, Hill WD, et al. Study of 300,486 individuals identifies 148 independent genetic loci influencing general cognitive function. Nat Commun 2018; 9(1): 2098

20 Shaffer JP. Multiple hypothesis testing. Ann Rev Psychol 1995; 46(1): 561-84.

21 Davison AC, Hinkley DV. Bootstrap Methods and Their Application. Cambridge University Press, 1997.

22 Purcell S, Neale B, Todd-Brown K, Thomas L, Ferreira MA, Bender D, et al. PLINK: a tool set for whole-genome association and population-based linkage analyses. Am J Hum Genet 2007; 81(3): 559-75.

23 Das S, Forer L, Schonherr S, Sidore C, Locke AE, Kwong A, et al. Next-generation genotype imputation service and methods. Nat Genet 2016; 48(10): 1284-7.

24 Loh P-R, Tucker G, Bulik-Sullivan BK, Vilhjálmsson BJ, Finucane HK, Salem RM, et al. Efficient Bayesian mixed model analysis increases association power in large cohorts. Nat Genet 2015; 47(3): 284-90.

25 Choi SW, Mak TSH, O'Reilly PF. Tutorial: a guide to performing polygenic risk score analyses. Nat Protoc 2020; 15: 2759-72.

26 ISHak WW, Mirocha J, James D, Tobia G, Vilhauer J, Fakhry H, et al. Quality of life in major depressive disorder before/after multiple steps of treatment and oneyear follow-up. Acta Psychiatr Scand 2015; 131(1): 51-60.

27 Skevington SM, Böhnke JR. How is subjective well-being related to quality of life? Do we need two concepts and both measures? Soc Sci Med 2018; 206 22-30.

28 Camfield L, Skevington SM. On subjective well-being and quality of life. J Health Psychol 2008; 13(6): 764-75.

29 Domenech C, Altamura C, Bernasconi C, Corral R, Elkis H, Evans J, et al. Healthrelated quality of life in outpatients with schizophrenia: factors that determine changes over time. Soc PSychiatry Psychiatr Epidemiol 2018; 53(3): 239-48.

30 Zheng J, Erzurumluoglu AM, Elsworth BL, Kemp JP, Howe L, Haycock PC, et al. LD Hub: a centralized database and web interface to perform LD score regression that maximizes the potential of summary level GWAS data for SNP heritability and genetic correlation analysis. Bioinformatics 2017; 33(2): 272-9.

31 Habtewold TD, Liemburg EJ, Islam MA, de Zwarte SMC, Boezen HM, Bruggeman $\mathrm{R}$, et al. Association of schizophrenia polygenic risk score with data-driven cognitive subtypes: a six-year longitudinal study in patients, siblings and controls. Schizophr Res 2020 (in press).

32 Chang CC, Chow CC, Tellier LC, Vattikuti S, Purcell SM, Lee JJ. Second-generation PLINK: rising to the challenge of larger and richer datasets. Gigascience 2015; $4: 7$

33 McLaughlin RL, Schijven D, van Rheenen W, van Eijk KR, O'Brien M, Kahn RS et al. Genetic correlation between amyotrophic lateral sclerosis and schizophrenia. Nat Commun 2017; 8: 14774.

34 Schur RR, Schijven D, Boks MP, Rutten BPF, Stein MB, Veldink JH, et al. The effect of genetic vulnerability and military deployment on the development of post-traumatic stress disorder and depressive symptoms. Eur Neuropsychopharmacol 2019; 29(3): 405-15.

35 Bulik-Sullivan B, Finucane HK, Anttila V, Gusev A, Day FR, Loh P-R, et al. An atlas of genetic correlations across human diseases and traits. Nat Genet 2015; 47 (11): 1236-41.

36 Purcell SM, Wray NR, Stone JL, Visscher PM, O'Donovan MC, Sullivan PF, et al. Common polygenic variation contributes to risk of schizophrenia and bipolar disorder. Nature 2009; 460(7256): 748-52.

37 Cruz BF, de Resende CB, Carvalhaes CF, Cardoso CS, Teixeira AL, Keefe RS, et al. Interview-based assessment of cognition is a strong predictor of quality of life in patients with schizophrenia and severe negative symptoms. Rev Bras Psiquiatr 2016; 38(3): 216-21.

38 Van Eck RM, Burger TJ, Vellinga A, Schirmbeck F, de Haan L. The relationship between clinical and personal recovery in patients with schizophrenia spectrum disorders: a systematic review and meta-analysis. Schizophr Bull 2018; 44(3): 631-42. 